\title{
Prognostic Value of Baseline Neutrophil-Lymphocyte and Platelet-Lymphocyte Ratios in Local and Advanced Gastric Cancer Patients
}

\author{
Mehmet Naci Aldemir ${ }^{1}$, Mehmet Turkeli ${ }^{1 *}$, Melih Simsek ${ }^{1}$, Nilgun Yildirim ${ }^{1}$, \\ Yusuf Bilen $^{2}$, Harun Yetimoglu ${ }^{3}$, Mehmet Bilici ${ }^{1}$, Salim Basol Tekin ${ }^{1}$
}

\begin{abstract}
Background: We aimed to investigate the prognostic value of baseline neutrophil, lymphocyte, and platelet counts along with the neutrophil-lymphocyte ratio (NLR) and platelet-lymphocyte ratio (PLR) in local and advanced gastric cancer patients. Materials and Methods: In this retrospective cross-sectional study, a total of 103 patients with gastric cancer were included. For all, patient characteristics and overall survival (OS) times were evaluated. Data from a complete blood count test including neutrophil, lymphocyte, monocyte, white blood cell (WBC) and platelet (PIt) count, hemoglobin level $(\mathrm{Hb})$ were recorded, and the NLR and PLR were obtained for every patient prior to pathological diagnosis before any treatment was applied. Results: Of the patients, 53 had local disease, underwent surgery and were administered adjuvant chemoradiotherapy where indicated. The remaining 50 had advanced disease and only received chemotherapy. OS time was $71.6 \pm 6$ months in local gastric cancer patients group and $15 \pm 2$ months in the advanced gastric cancer group. Univariate analysis demonstrated that only high platelet count $(p=0.013)$ was associated with better $O S$ in the local gastric cancer patients. In contrast, both low NLR ( $p=0.029)$ and low PLR $(p=0.012)$ were associated with better OS in advanced gastric cancer patients. Conclusions: This study demonstrated that NLR and PLR had no effect on prognosis in patients with local gastric cancer who underwent surgery and received adjuvant chemoradiotherapy. In advanced gastric cancer patients, both NLR and PLR had significant effects on prognosis, so they may find application as easily measured prognostic factors for such patients.
\end{abstract}

Keywords: Gastric cancer - neutrophil lymphocyte ratio - platelet lymphocyte ratio - prognosis

Asian Pac J Cancer Prev, 16 (14), 5933-5937

\section{Introduction}

Gastric cancer is the fourth most common cancer and the second leading cause of cancer-related death worldwide (Herszenyi et al., 2010). Because the disease is frequently asymptomatic at early stages, it usually diagnoses at advanced stages (Maconi et al., 2008). Chemotherapy in metastatic gastric cancer patients can improve survival and quality of life, however median overall survival still remains often less than 1 year together with significant side effects (Oba et al., 2013). The causes of poor prognosis are associated with both tumor-related and host-related factors, especially the systemic inflammatory response (Colotta et al., 2009).

Systemic inflammatory response to tumors increases metastasis through the inhibition of apoptosis, augmentation of angiogenesis, and DNA damage (Jackson et al., 1997; Jaiswal et al., 2000; Balkwill et al., 2001; Coussens et al., 2002). It was observed that inflammatory cells accumulating around tumor cells significantly effects the progression of the tumor and the prognosis.
When there is lymphocyte infiltration around the tumor, the prognosis of the patient is better than those without infiltration (Kawata et al., 1992). On the contrary, tissue neutrophilia in the stroma of tumor may lead to poor prognosis (Yamashita et al., 1995). Increases in neutrophil counts and decreases in lymphocytes counts according to the systemic inflammatory response during the cancer progression process were also reported (Satomi et al., 1995). It is well known that systemic inflammatory response is closely associated with the progression of cancer. The invasion and metastasis ability of a tumor is dependent on both the intrinsic characteristics of the tumor cells and the tumor microenvironment (Balkwill et al., 2001; Coussens et al., 2002). In various types of malignancies inflammatory parameters like the modified Glasgow Prognostic Score (mGPS), C-reactive protein (CRP), number of leukocyte, neutrophil, monocyte, lymphocyte, and platelets, neutrophil to lymphocyte ratio (NLR), and platelet to lymphocyte ratio (PLR), have been recommended as prognostic factors (Yamanaka et al., 2007; Li et al., 2014).

${ }^{1}$ Department of Medical Oncology, ${ }^{2}$ Department of Hematology, ${ }^{3}$ Department of Internal Medicine, Faculty of Medicine, Ataturk University,Erzurum,Turkey*For correspondence:dr.mturkeli@mynet.com 
In gastric cancer some serum biomarkers are associated with poor prognosis, but they are not useful because of cost and time-consuming (Wurl et al., 1997; Nakajima et al., 1998). However, currently novel immunological and histological biomarkers have been identified (Bao et al., 2010; Shirai et al., 2010; Kim et al., 2011). It was reported that a high NLR was an independent predictor factor of poor survival in patients with advanced gastric cancer treated with chemotherapy (Li et al., 2014). NLR also was found to be associated with survival in lung, bladder, gastric, pancreatic, colorectal and ovarian cancers (Walsh et al., 2005; Liu et al., 2010; Proctor et al., 2011; Unal et al., 2013). Like neutrophil and lymphocyte counts, platelet counts play an important role in prognosis of malignancies. An increase in platelet counts leads tumor progression by enhancing angiogenesis. PLR is suggested to be associated with survival in pancreas, lung and breast cancer. NLR and PLR also were found as predictors for cancer specific survival in renal cancer, gynecological, colorectal, bladder, gastroesophageal, breast, prostatic, and lung cancer (Proctor et al., 2011).

The aim of this study was to evaluate the prognostic significance of pre-chemotherapy and pre-operative NLR and PLR in local and metastatic gastric cancer and to permit more accurate patient stratification by the way improving clinical decision.

\section{Materials and Methods}

This retrospective cross-sectional study was conducted between the years 2006-2013 in the Medical Oncology Department of Ataturk University Faculty of Medicine in Erzurum, Turkey. A total of 103 patients who were diagnosed with gastric cancer were included. Gastric cancer diagnosis was established with imaging methods and histopathological evaluation of endoscopic biopsy materials. Of the patients, 53 had local disease, undergone surgery and were administered adjuvant chemoradiotherapy who were indicated. The other 50 patients had advanced disease and they only received chemotherapy.

Patients with diseases that can effect NLR and PLR including secondary or priory malignancies, hematological diseases, inflammatory diseases, priory chemotherapy history were excluded.

For all patients; gender, age, Eastern Cooperative Oncology Group (ECOG) performance status, disease stage, and overall survival times were evaluated. An available complete blood count (CBC) test was obtained from every patient prior to pathological diagnosis before any treatments were applied. CBC parameters including neutrophil, lymphocyte, monocyte, white blood cell (WBC) and platelet (Plt) count, hemoglobin (Hb) level were recorded, and the NLR and PLR were calculated.

Blood samples analyzed by full automatic hematology analyzer named Beckman Coulter LH 780 machine. WBC and dependent tests were measured according to volume, conductivity, and laser scatter basis; Plt was measured with electrical impedance method.

NLR was defined as the ratio of absolute neutrophil count to absolute lymphocyte count; PLR was defined as the ratio of absolute platelet count to absolute lymphocyte count. In previous studies, a NLR cut-off value was used between 2.5-5.0 (Walsh et al., 2005; Yamanaka et al., 2007; Gomez et al., 2008; Aliustaoglu et al., 2010; Shimada et al., 2010; Unal et al., 2013) and a PLR cutoff value was used between 150-300 (Aliustaoglu et al., 2010; Unal et al., 2013; Templeton et al., 2014).We used the medians of distribution as the cut-off values. NLR was categorized into two groups $(<2.75$ and $\geq 2.75)$, and PLR was also categorized into two groups $(<170$ and $\geq 170)$.

\section{Statistical analysis}

For statistical analysis SPSS version 20.0 package program (Chicago,IL, USA) was used. Overall survival (OS) time was calculated from date of diagnosis to date of death due to any reason. Univariate survival analysis was performed using the Kaplan-Meier method with the log-rank test to analyze patient outcome. $\mathrm{p}<0.05$ was considered as statistically significant.

\section{Results}

\section{Patient characteristics}

There were 103 patients with the diagnosis of gastric cancer who have satisfied inclusion criteria. Among local gastric cancer patients, there were 53 patients $28(52.8 \%)$ of whom were male and $25(47.2 \%)$ were female. Mean age was 58 years (range 28-78 years).OS time was $71.6 \pm 6$ months (Figure 1).Twenty three $(43.4 \%)$ patients were ECOG 0, 25 (47.2\%) were ECOG 1, and $5(9.4 \%)$ were ECOG 2. Of the patients, 19 (35.8\%) were died during follow up.

In advanced gastric cancer patients, there were 50 patients $30(60 \%)$ of whom were male and $20(40 \%)$ were female. Mean age was 65 years (range $40-82$ years). OS time was $15 \pm 2$ months (Figure 1). Eight patients (16\%) were ECOG 0, $34(68 \%)$ were ECOG 1, and $8(16 \%)$ were ECOG 2. Of the patients $41(82 \%)$ were died during follow up.

\section{Systemic inflammatory response variables}

We evaluated the neutrophil, lymphocyte, and platelet counts of our patients. The median values of NLR and PLR were 2.75 and 170, respectively. There were 52 (50.5\%) patients who had NLR $\geq 2.75$ and $51(49.5 \%)$ patients who had $P L R \geq 170$.

In local gastric cancer patients group, $8(15.1 \%)$ patients had an elevated neutrophil count, $8(15.1 \%)$ patients had an elevated platelet count, and $3(5.7 \%)$ patients had a decreased lymphocyte count. Among advanced gastric cancer patients, $11(22 \%)$ patients had an elevated neutrophil count, 21 (42\%) patients had an elevated platelet count, and $4(8 \%)$ patients had a decreased lymphocyte count.

\section{Effect of systemic inflammatory response variables on OS}

The relationship between the demographical features, systemic inflammatory response variables, and OS according to the local or advanced disease status of the patients is demonstrated in Table 1. The univariate analysis demonstrated that only high platelet count was associated 

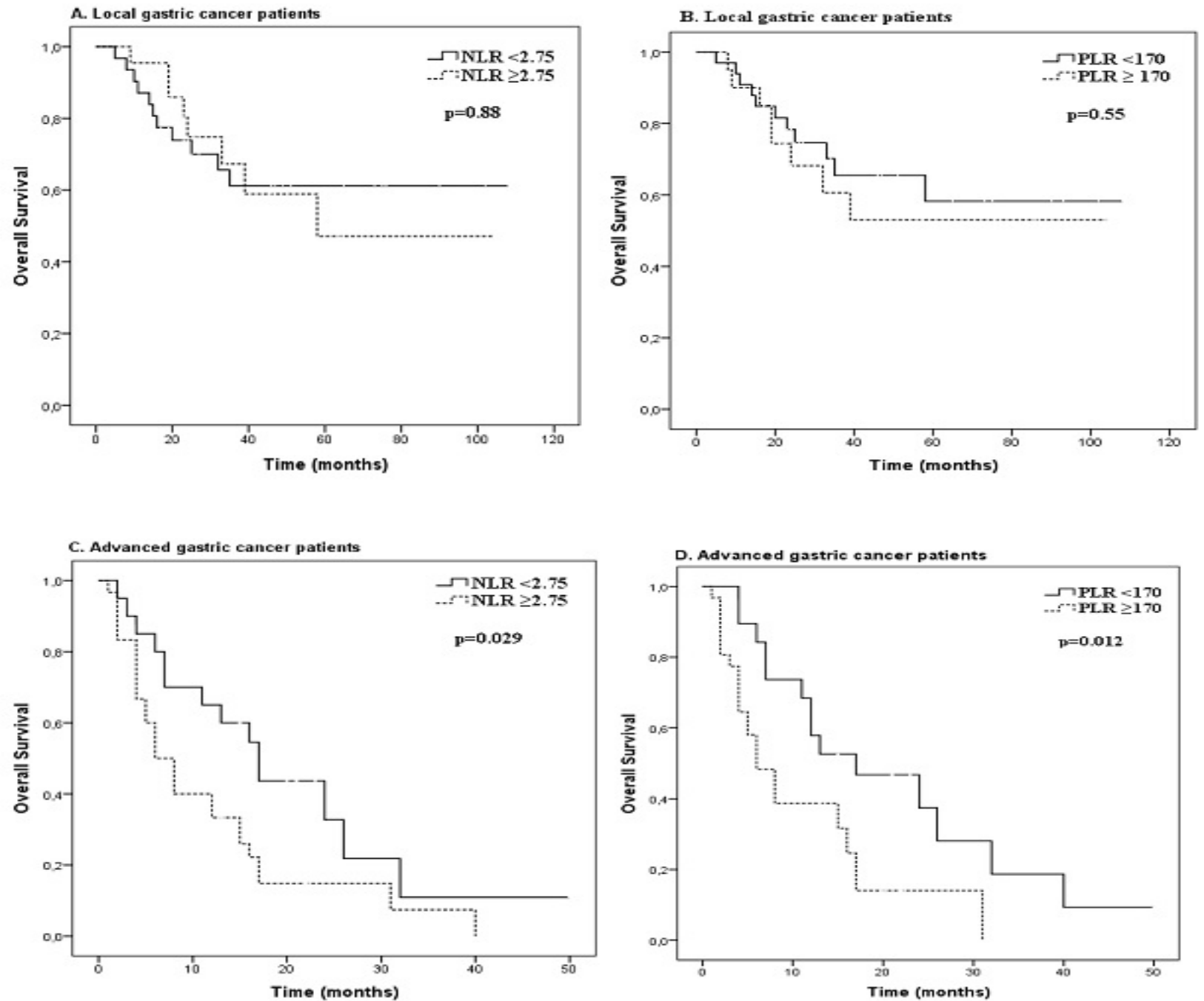

Figure 1. Overall Survival Times of Patients According to NLR and PLR. (A) OS according to NLR in local gastric cancer patients group; (B) OS according to PLR in local gastric cancer patients group; (C) OS according to NLR in advanced gastric cancer patients group; (D) OS according to PLR in advanced gastric cancer patients group

Table 1. The Relationship between the Demographical Features, Systemic Inflammatory Response Variables, and OS According to the Local or Advanced Disease Status of the Patients

\begin{tabular}{|c|c|c|c|c|c|c|c|}
\hline & & \multicolumn{3}{|c|}{ LocalGastricCancer $(n=53)$} & \multicolumn{3}{|c|}{ Advanced GastricCancer $(\mathrm{n}=50)$} \\
\hline & & No. of patients (\%) & OS (months) & p-value & No. of patients $(\%)$ & OS (months) & p-value \\
\hline \multirow[t]{2}{*}{ Gender } & Male & $28(52.8)$ & $67.8 \pm 9$ & 0.32 & $30(60)$ & $15.4 \pm 2$ & 0.66 \\
\hline & Female & $25(47.2)$ & $74.6 \pm 8$ & & $20(40)$ & $14.4 \pm 4$ & \\
\hline \multirow[t]{3}{*}{ ECOG } & 0 & $23(43.4)$ & $87.6 \pm 8$ & 0.001 & $8(16)$ & $30.1 \pm 4$ & 0.002 \\
\hline & 1 & $25(47.2)$ & $57.4 \pm 8$ & & $34(68)$ & $13 \pm 2$ & \\
\hline & 2 & $5 \quad(9.4)$ & $18.8 \pm 6$ & & $8(16)$ & $8 \pm 4$ & \\
\hline \multirow[t]{2}{*}{ Age } & $\leq 65$ & $40(75.5)$ & $73.4 \pm 7$ & 0.49 & $29(58)$ & $14.2 \pm 2$ & 0.75 \\
\hline & $>65$ & $13(24.5)$ & $49.6 \pm 9$ & & $21(42)$ & $16 \pm 4$ & \\
\hline \multirow[t]{2}{*}{ NLR } & $<2.75$ & $31(58.5)$ & $73.3 \pm 8$ & 0.88 & $20(40)$ & $19.8 \pm 4$ & 0.029 \\
\hline & $\geq 2.75$ & $22(41.5)$ & $66.4 \pm 10$ & & $30(60)$ & $11.7 \pm 2$ & \\
\hline \multirow[t]{2}{*}{ PLR } & $<170$ & $33(62.3)$ & $74.2 \pm 8$ & 0.55 & $19(38)$ & $21.1 \pm 4$ & 0.012 \\
\hline & $\geq 170$ & $20(37.7)$ & $65.7 \pm 10$ & & $31(62)$ & $11 \pm 2$ & \\
\hline \multirow[t]{2}{*}{ Neutrophil count } & $<8 \times 109 / 1$ & $45(84.9)$ & $71 \pm 7$ & 0.5 & $39(78)$ & $14.5 \pm 2$ & 0.55 \\
\hline & $\geq 8 \times 109 / 1$ & $8(15.1)$ & $53.2 \pm 6$ & & $11(22)$ & $12.8 \pm 5$ & \\
\hline \multirow[t]{2}{*}{ Lymphocyte count } & $<1 \times 109 / 1$ & $3(5.7)$ & $45.7 \pm 15$ & 0.8 & $4(8)$ & $8.3 \pm 4$ & 0.44 \\
\hline & $\geq 1 \times 109 / 1$ & $50(94.3)$ & $71.7 \pm 7$ & & $46(92)$ & $15.3 \pm 2$ & \\
\hline \multirow[t]{2}{*}{ Platelet count } & $<350 \times 109 / 1$ & $45(84.9)$ & $77 \pm 7$ & 0.013 & $29(58)$ & $16.2 \pm 3$ & 0.33 \\
\hline & $\geq 350 \times 109 / 1$ & $8(15.1)$ & $24.1 \pm 3$ & & $21(42)$ & $12.8 \pm 3$ & \\
\hline \multirow[t]{2}{*}{ Last status } & Alive & $34(64.2)$ & & & $9(18)$ & & \\
\hline & Dead & $19(35.8)$ & & & $41(82)$ & & \\
\hline
\end{tabular}


with better OS in local gastric cancer patients group. Contrarily both low NLR and low PLR were associated with better OS in advanced gastric cancer patients group.

\section{Discussion}

Gastric cancer is a common cancer and one of the frequent causes of cancer-related death worldwide (Herszenyi et al., 2010). Advanced stage gastric cancer has a poor prognosis and various factors effect this situation. One of the most important factors among these is systemic inflammatory response including neutrophil to lymphocyte ratio (NLR), and platelet to lymphocyte ratio (PLR). Although underlying mechanism is unclear, recent studies reported that elevated pretreatment peripheral blood neutrophil, and platelet counts have been associated with poor survival in several cancers. Similarly, in our study, both in local and advanced gastric cancer patients OS was shorter with higher neutrophil and platelet counts, but in each two groups the difference did not reach statistical significance.

The prognostic significance of an increased NLR has been determined in colorectal cancer, intrahepatic cholangiocarcinoma, hepatocellular carcinoma, and pancreatic cancer (Walsh et al., 2005; Gomez et al., 2008a; 2008b; An et al., 2010; Liu et al., 2010). Some studies have shown that a high pre-operative NLR has been a useful predictor of survival in advanced non-smallcell lung cancer, colorectal cancer, and gastric cancer (Walsh et al., 2005; Teramukai et al., 2009; Ding et al., 2010). In a study, NLR was revealed as an independent predictor of overall survival in relatively early staged operable gastric cancer (Mohri et al., 2010). Also the preoperative NLR was associated with survival in late stage gastric cancer patients who undergone potentially curative resection (Jung et al., 2011). Among 8759 cancer patients, it was shown that the systemic inflammationbased scores have prognostic value (Proctor et al., 2011). Of all these inflammatory parameters, especially NLR has been reported to be a prognostic factor in gastric cancer (Hirashima et al., 1998; Yamanaka et al., 2007; Aliustaoglu et al., 2010; Shimada et al., 2010; Jung et al., 2011). High NLR was associated with poor OS in early and late stage gastric cancer. In our study, we found that in local gastric cancer patients higher NLR levels ( NLR $\geq 2.75$ ) were associated with a shorter survival time, but the difference was not statistically significant. On the contrary, in advanced gastric cancer patients, higher NLR levels were associated with a shorter survival time and the difference was statistically significant. Similarly to our findings, Yamanaka et al. were reported significant correlation between NLR and survival in stage IV gastric cancer patients (Yamanaka et al., 2007).

Platelets may have an important role in tumor growth by inducing angiogenesis via vascular endothelial growth factor (VEGF) (Kusumanto et al., 2003; Kassim et al., 2004). In a meta-analysis including 12,754 solid tumor patients and including twenty studies, the prognostic role of PLR was evaluated. They found that there was an association between elevated PLR and poor survival. This association was observed in various solid tumors and both for metastatic and non-metastatic disease. But they found the strongest association between PLR and survival in metastatic disease when compared with locoregional disease (Templeton et al., 2014). Also an association between the preoperative platelet count and survival was observed in resected pancreatic adenocarcinoma (Schwarz et al., 2001; Suzuki et al., 2004; Brown et al., 2005). But there is controversy on the effect of high platelet counts to survival outcome. Contrarily it was observed that in other gastrointestinal malignancies increased platelet counts were associated with poorer survival (Monreal et al., 1998; Schwarz et al., 2001; Suzuki et al., 2004; Brown et al., 2005; Shimada et al., 2010). In evaluation of an ovarian mass, Topcu et al. (2014) have found that PLR may be a prognostic factor, while NLR is ineffective marker. Pretreatment NLR and PLR measurements were suggested as important prognostic factors and evaluation of these two parameters may predict a better prognosis in NSCLC patients (Unal et al., 2013). In our study, we found that in local gastric cancer patients, survival time was shorter with higher PLR levels (PLR $\geq 170$ ), but the difference was not statistically significant. On the contrary, in advanced gastric cancer patients, survival time was shorter with higher PLR levels and the difference was statistically significant.

Limitations of our study are its retrospective design and low number of patients. Further well-designed prospective studies including large number of gastric cancer patients are required. Moreover, whether the cutoff value of 2.75 for NLR and 170 for PLR are correct requires further investigation.

In conclusion, peripheral blood sampling is a routinely used procedure and samples are easily obtained from patients. While NLR and PLR did not show prognosis in patients with local gastric cancer who underwent surgery and received adjuvant chemoradiotherapy, in advanced gastric cancer patients both NLR and PLR may give clue for the prognosis.

\section{References}

Aliustaoglu M, Bilici A, Ustaalioglu BB, et al (2010). The effect of peripheral blood values on prognosis of patients with locally advanced gastric cancer before treatment. Med Oncol, 27, 1060-5.

An X, Ding PR, Li YH, et al (2010). Elevated neutrophil to lymphocyte ratio predict ssurvival in advanced pancreatic cancer. Biomarkers, 15, 516-22.

Balkwill F, Mantovani A (2001). Inflammation and cancer: BacktoVirchow? Lancet, 357, 539-45.

Bao GQ, Qiao Q, Zhao HD, et al (2010). Prognostic value of HMGB1 overexpression in resectable gastric adenocarcinomas. World J Surg Oncol, 8, 52.

Brown KM, Domin C, Aranha GV, et al (2005). Increased preoperative platelet count is associated with decreased survival after resection for adenocarcinoma of the pancreas. Am J Surg, 189, 278-82.

Colotta F, Allavena P, Sica A, et al (2009). Cancer-related inflammation, the seventh hallmark of cancer: links to genetic instability. Carcinogenesis, 30, 1073-81.

Coussens LM, Werb Z (2002). Inflammation and cancer. Nature, 420, 860-7.

Ding PR, An X, Zhang RX, et al (2010). Elevated preoperative 
Prognostic Value of Neutrophil-Lymphocyte and Platelet-Lymphocyte Ratios in Local and Advanced Gastric Cancer Patients neutrophil to lymphocyte ratio predicts risk of recurrence following curative resection for stage IIA colon cancer. Int J Colorectal Dis, 25, 1427-33.

Gomez D, Farid S, Malik HZ, et al (2008a). Preoperative neutrophil to lymphocyte ratio as a prognostic predictor after curative resection for hepatocellular carcinoma. World J Surg, 32, 1757-62.

Gomez D, Morris-Stiff G, Toogood GJ, et al (2008b). Impact of systemic inflammation on outcome following resection for intrahepatic cholangiocarcinoma. J Surg Oncol, 97, 513-8.

Herszenyi L, Tulassay Z (2010). Epidemiology of gastrointestinal and liver tumors. Eur Rev Med Pharmacol Sci, 14, 249-58.

Hirashima M, Higuchi S, Sakamoto K, et al (1998). The ratio of neutrophils to lymphocytes and the phenotypes of neutrophils in patients with early gastric cancer. J Canc Res Clin Oncol, 124, 329-34.

Jackson JR, Seed MP, Kircher CH, et al (1997). The codependence of angiogenesis and chronic inflammation. FASEB $J, \mathbf{1 1}$, 457-65.

Jaiswal M, LaRusso NF, Burgart LJ, et al (2000). Inflammatory cytokines induce DNA damage and inhibit DNA repair in cholangiocarcinoma cells by a nitric oxide-dependent mechanism. Cancer Res, 60, 184-90.

Jung MR, Park YK, Jeong O, et al (2011). Elevated preoperative neutrophil to lymphocyte ratio predicts poor survival following resection in late stage gastric cancer.J Surg Oncol, 104, 504-10.

Kassim SK, El-Salahy EM, Fayed ST, et al (2004). Vascular endothelial growth factor and interleukin- 8 are associated with poor prognosis in epithelial ovarian cancer patients. Clin Biochem, 37, 363-9.

Kawata A, Une Y, Hosokawa M, et al (1992). Tumor-infiltrating lymphocytes and prognosis of hepatocellular carcinoma. Jpn J Clin Oncol, 22, 256-63.

Kim KH, Kwon HC, Oh SY, et al (2011). Clinicopathological significance of ERCC1, thymidylate synthase and glutathione S-transferase P1 expression for advanced gastric cancer patients receiving adjuvant 5 -Fu and cisplatin chemotherapy. Biomarkers, 16, 74-82.

Kusumanto YH, Dam WA, Hospers GA, et al (2003). Platelets and granulocytes, in particular the neutrophils, form important compartments for circulating vascular endothelial growth factor. Angiogenesis, 6, 283-7.

Li QQ, Lu ZH, Yang L, et al (2014). Neutrophil count and the inflammation-based glasgow prognostic score predict survival in patients with advanced gastric cancer receiving first-line chemotherapy. Asian Pac J Cancer Prev, 15, 945-50.

Liu H, Liu G, Bao Q, et al (2010). The baseline ratio of neutrophil to lymphocytes is associated with patient prognosis in rectal carcinoma. J Gastrointest Cancer, 41, 116-20.

Maconi G, Manes G, Porro GB (2008). Role of symptoms in diagnosis and outcome of gastric cancer. World $J$ Gastroenterol, 14, 1149-55.

Mohri Y, Tanaka K, Ohi M, et al (2010). Prognostic significance of host-and tumor-related factors in patients with gastric cancer. World J Surg, 34, 285-90.

Monreal M, Fernandez-Llamazares J, Pinol M, et al (1998). Platelet count and survival in colorectal cancer-a preliminary study. Thromb Haemost, 79, 916-8.

Nakajima K, Ochiai T, Suzuki T, et al (1998). Impact of preoperative serum carcino embryonic antigen, CA 19-9 and alpha feto protein levels in gastric cancer patients. Tumour Biol, 19, 464-9.

Oba K, Paoletti X, Bang YJ, et al (2013). Role of chemotherapy for advanced /recurrent gastric cancer: an individual-patientdata meta-analysis. Eur J Cancer, 49, 1565-77.

Proctor M, Morrison D, Talwar D, et al (2011). A comparison of inflammation-based prognostic scores in patients with cancer. A Glasgow inflammation outcome study. Eur $J$ Cancer, 47, 2633-41.

Satomi A, Murakami S, Ishida K, et al (1995). Significance of increased neutrophils in patients with advanced colorectal cancer. Acta Oncol, 34, 69-73.

Schwarz RE, Keny H (2001). Preoperative platelet count predicts survival after resection of periampullary adenocarcinoma. Hepatogastroenterology, 48, 1493-8.

Shimada H, Tajiguchi N, Kainuma O, et al (2010). High preoperative neutrophil lymphocyte ratio predicts poor survival in patients with gastric cancer. Gastric Canc, 13, 170-6.

Shirai O, Ohmiya N, Taguchi A, et al (2010). P53, P21 and P73 gene polymorphisms in gastriccarcinoma. Hepatogastroenterology, 57, 1595-601.

Suzuki K, Aiura K, Kitagou M, et al (2004). Platelet counts closely correlate with the disease-free survival interval of pancreatic cancer patients. Hepatogastroenterology, 51, 847-53.

Templeton AJ, Ace O, McNamara MG, et al (2014). Prognostic role of platelet to lymphocyte ratio in solid tumors: a systematic review and meta-analysis. Cancer Epidemiol Biomarkers Prev, 23, 1204-12.

Teramukai S, Kitano T, Kishida Y, et al (2009). Pretreatment neutrophil count as an independent prognostic factor in advanced non-small-cell lung cancer: an analysis of Japan multinational trial organisation LC00-03. Eur J Canc, 45, 1950-8.

Topcu HO, Guzel AI, Ozer I, et al (2014). Comparison of neutrophil/lymphocyte and platelet/ lymphocyte ratios for predicting malignant potential of suspicious ovarian masses in gynecology practice. Asian Pac J Cancer Prev, 15, 6239-41.

Unal D, Eroglu C, Kurtul N, et al (2013). Are neutrophil/ lymphocyte and platelet/lymphocyte rates in patients with non-small cell lung cancer associated with treatment response and prognosis? Asian Pac J Cancer Prev, 14, 5237-42.

Walsh SR, Cook EJ, Goulder F, et al (2005). Neutrophillymphocyte ratio as a prognostic factors in colorectal cancer. J Surg Oncol, 91, 181-4.

Würl P, Weigmann F, Meye A, et al (1997). Detection of p53 autoantibodies in sera of gastric cancer patients and their prognostic relevance. Scand J Gastroenterol, 32, 1147-51.

Yamanaka T, Matsumoto S, Teramukai S, et al (2007). The baseline ratio of neutrophils to lymphocytes is associated with patient prognosis in advanced gastric cancer. Oncology, 73, 215-20

Yamashita J, Ogawa M, Shirakusa T (1995). Free-from neutrophil elastase is an independent marker predicting recurrence in primary breast cancer. J Leukoc Biol, 57, 375-8. 